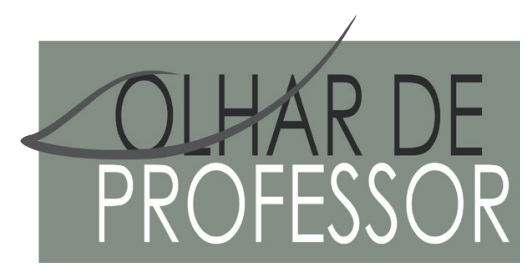

DOl: 10.5212/OLHARPROFR.v.23.2020.15510.209209225352.0521

\title{
CONVIIÊNCIA VIRTUALIZADA ENTRE ESCOLA E LAR EM TEMPOS DE PANDEMIA: UMA REFLEXÃo SÓCIO-EDUCATIVA
}

\author{
VIRTUALIZED COEXISTENCE BETWEEN SCHOOL AND HOME IN TIMES OF PANDEMIC: A \\ SOCIOPEDAGOGICAL REFLECTION
}

\author{
LA CONVIVENCIA VIRTUALIZADA ENTRE LA ESCUELA Y EL HOGAR EN TIEMPOS DE PANDEMIA: UNA \\ REFLEXIÓN SOCIOPEDAGÓGICA
}

PABLO NAHUEL DI NAPOLI

\begin{abstract}
Resumo: Este 2020 estamos vivendo um momento excepcional causada por uma pandemia (COVID-19) que, apesar de haver sido escrita em roteiros de cinema, nunca havíamos enfrentado. Vivenciamos um distanciamento sociocorporal, enquadrado em uma política global de quarentena preventiva, que tem perturbado instituições sociais, estilos de vida e sensibilidades subjetivas. Uma dessas instituições é a escola, que pela primeira vez em sua história foi forçada a fechar suas portas físicas inoportunamente. O objetivo deste ensaio é pensar nestes tempos sobre a reconfiguração da convivência espaço-tempo entre escola e lar com base em três aspectos: temporalidade escolar, formato escolar e contextos de desigualdade educacional. É uma reflexão analítica que recupera depoimentos escola da pesquisa qualitativa e um site bem-humorado. A conclusão é que a ausência da materialidade da escola torna seu potencial ainda mais visível.
\end{abstract}

Palavras-chave: Temporalidade escolar; forma escolar; profissão docente; desigualdade.

\begin{abstract}
This 2020 we are living in an exceptional time caused by a pandemic (COVID-19) that, although it has been written in film scripts, we had never confronted. We experienced a socio-corporal distancing framed in a global policy of preventive quarantine that has disrupted social institutions, lifestyles and subjective sensibilities. One of these institutions is the school, which for the first time in its history has been forced to close its physical doors inopportunely. In this short The aim of this essay is to think in these times about the reconfiguration of the space-time coexistence between school and home based on three axes: school temporality, school format and contexts of educational inequality. It is an analytical reflection that recovers school testimonies from qualitative research and a humorous web page. It concludes that the absence of the materiality of schooling makes its potential even more visible.
\end{abstract}

Keywords: Temporary schooling; school format; teaching profession; inequality.

Resumen: Este 2020 vivimos un tiempo de excepción provocado por una pandemia (COVID-19) que, por más que haya sido escrita en guiones cinematográficos, nunca la habíamos afrontado. Experimentamos un distanciamiento sociocorporal, enmarcado en una política mundial de cuarentena preventiva, que ha trastocado las instituciones sociales, los estilos de vida y las sensibilidades subjetivas. Una de dichas instituciones es la escuela, que por primera vez en su historia se ha visto forzada a cerrar intempestivamente sus puertas físicas. Este ensayo tiene como objetivo pensar en estos tiempos la reconfiguración de la convivencia espacio-temporal entre la escuela y el hogar a partir de tres ejes: la temporalidad escolar, el formato escolar y los contextos de desigualdad educativa. Se

\footnotetext{
* Doctor en Ciencias Sociales y Profesor y Licenciado en Sociología por la Universidad de Buenos Aires, Argentina. Actualmente se desempeña como becario del Programa de Becas Posdoctorales en la Universidad Nacional Autónoma de México con sede en el Instituto de Investigaciones sobre la Universidad y la Educación (IISUE), asesorado por la Dra. Leticia Pogliaghi. E-mail: pablodinapoli@filo.uba.ar.
} 
trata de una reflexión analítica que recupera testimonios escolares de una investigación cualitativa y de una página web humorística. Se concluye que la ausencia de la materialidad de la escuela visibiliza aún más su potencia.

Palabras-clave: Temporalidad escolar; formato escolar; oficio docente; desigualdad.

\section{INTRODUCCIÓN}

Durkheim (1987) decía algo así como que la fuerza de los hechos sociales puede sentirse y percibirse cuando se intenta ir a contracorriente de ellos. Pues en este contexto excepcional donde la rutina mundial se ha trastocado, se ocupe la posición social que se ocupe, es un momento propicio para empezar a "tocar" algunas de esas cosas llamadas hechos sociales.

La pregunta que estructura el presente ensayo analítico es: ¿Cómo se reconfigura la convivencia espacio-temporal de la escuela y el hogar en tiempos de coronavirus? A partir de una serie de testimonios y escenas extraídas de una investigación cualitativa en curso y de un canal de youtube humorístico, en este texto reflexionamos teóricamente, en primer lugar, sobre el trastocamiento de la temporalidad en los espacios hogareños en contextos de desigualdad; en segundo lugar, respecto de la resignificación del oficio docente pero bajo un formato escolar incorporado que se nos hace omnipresente y; en tercer lugar, abordamos cómo la virtualización forzada de la educación requiere de cierto autocontrol individual por parte de niñas, niños y jóvenes. Por último, a modo de conclusión, sostenemos que la ausencia de la materialidad de la escuela visibiliza aún más su potencia social y pedagógica.

\section{TRASTOCAMIENTODE LA TEMPORALIDADESCOLAR}

Sabemos, el tiempo de la escuela no solo rige la temporalidad del ámbito escolar sino también la familiar y marca la biografía de cada uno de sus integrantes. Es decir que la temporalidad escolar va más allá de la escuela. También es cierto que dicha temporalidad afecta de modo desigual las experiencias individuales y familiares en función de la clase, el género y el origen étnico. Los sectores sociales de mayores recursos económicos y culturales tienen más posibilidades de estructurar sus biografías con cuestiones escolares o valoradas por la escuela (doble escolaridad, escuelas con alta dotación tecnológica, actividades extracurriculares). En cambio en los sectores sociales de menores ingresos el tiempo escolar ocupa un porcentaje menor en sus temporalidades vitales, hay otras cuestiones que le compiten (el tiempo para llegar a la escuela, las tareas hogareñas, el trabajo). Esto lo observamos claramente tanto entre estudiantes de escuelas secundarias de la Ciudad Autónoma de Buenos Aires (CABA) como en estudiantes de bachillerato de la Ciudad de México. Es lo que Gonzalo Saraví (2015) llama escuela total y escuela acotada en el marco de la fragmentación social que atraviesa la experiencia de lxs jóvenes.

La escuela no se limita a la enseñanza y aprendizaje de contenidos curriculares, sino que también interviene en la estructuración de tiempos, espacios, comportamientos y personalidades, construye subjetividad. El orden del tiempo y el espacio forman parte de una coacción civilizadora. Sin embargo, hoy cuando se habla de "continuidad pedagógica" se pone el foco solo en uno de los aspectos escolares: la continuidad, cueste lo que cueste y caiga quien caiga, de la enseñanza y el aprendizaje de los contenidos prescriptos en la currícula.

Con la pandemia del COVID-19 y la cuarentena forzada en varios países no desapareció la escuela, pero si se trastocaron dos de sus pilares fundamentales: la arquitectura y el calendario escolar. Como dice Escolano (2000, p.40) "los cronosistemas del almanaque y el horario encuentran su soporte y acomodo en los escenarios físicos que dan cobertura y viabilidad a los tiempos y movimientos”. Desde ya que la relación de estos dos pilares veían en tensión y modificándose al son de las transformaciones estructurales del último siglo, lo que Martín Barbero (2008) definió, por ejemplo, como el pasaje de una sociedad con sistema educativo a una sociedad educativa. Sabemos, el modelo escolar fue rebasado tanto espacial como temporalmente por otros procesos de formación característicos de la era informacional. Ahora bien, el edificio escolar y los recreos estaban, rebasados pero estaban, se podía "tocar". 
Al desaparecer el escenario físico se trastocó el cronosistema escolar, familiar, laboral y de ocio. Se podría decir que adquirió mayor preponderancia el tiempo sobre el espacio. De hecho, una de las (pre) ocupaciones de los padres, de aquellos que disponen de tiempo, radica en cómo construir los espacios para el tiempo de la virtualidad escolarizada. A su vez, esta supremacía del tiempo sin espacio definido o con “espacios flexibles”, acelera lo que Escolano ya hace 20 años llamaba alienación educativa. Hoy día, sin salir de sus hogares, todos los actores de la comunidad educativa expresan estar agotados. ¿Qué está pasando con nuestra corporalidad que moviéndonos menos estamos más cansados?

Ahora parece ser la familia la responsable de construir la arquitectura escolar. Esa demanda de acompañamiento y compromiso que la escuela le pidió históricamente a la familia se hace presente, y su ausencia más notoria. La desigualdad, esa que la escuela ayuda a consolidar pero que también combate, se agranda y/o visibiliza aún más. Justamente el hecho de que la clausura física de la escuela (nunca espiritual y simbólica) haga más notoria la desigualdad, es una certeza para sostener que la escuela no solo la reproduce sino que también la atenúa e, incluso, brinda espacios para combatirla, aunque sea en su ideario.

Nos preguntamos qué pasa cuando los padres no pueden hacerse cargo de esa responsabilidad. Aquellos padres que no están en la casa porque no pueden darse el lujo de encuarentenarse ya sea porque tienen que ir a trabajar para "ganarse el pan" o hacer largas colas para conseguirlo. O las casas donde están los padres pero no hay espacio (una habitación, una mesa para...), materiales (un libro, una lapicera, una computadora, una hora para...) un tiempo (un silencio, un momento para...). O los padres que están absorbidos por sus trabajos, que en algunos casos consiste en producir clases virtuales para otros hijos, y no disponen de tiempo suficiente para gestionar la temporalidad escolar de sus hijos e hijas ${ }^{1}$. La arquitectura escolar clásica, decimonónica, ese antro de encierro y disciplinamiento social y subjetivo, le brinda a muchos niñas, niños y jóvenes la posibilidad de tener un espacio, un tiempo y una persona adulta que le cuida, que no tendrían de otro modo. Se trata de un espacio-tiempo de enseñanza y aprendizaje que va más allá del contenido curricularmente prescripto, se trata de la posibilidad de vivir una experiencia escolar que de otro modo sería diferente o no sería escolar.

\section{RESIGNIFICACIÓN DEL OFICIO DOCENTE}

Pensemos en las madres y los padres que tienen disponibilidad para construir/propiciar cierta temporalidad escolar, sea del modo que sea. Uno de los sentimientos que aparecen es el temor a no tener el suficiente capital cultural, conocimiento y/o ese saber pedagógico, muchas veces simplificado eufemísticamente en el término "paciencia”, para acompañar a sus hijos. En México, de la Cruz Flores (2020, p.44) sostiene que las principales dificultades que expresan las familias hoy para apoyar a sus hijos en las tareas escolares son: “a) no contar con estrategias para favorecer el aprendizaje; b) dificultades para expresarse, y c) poca comprensión sobre los métodos que utilizan los profesores en clase”.

El temor de los padres, producto del amor hacia los otros, nuestros hijos, nos brinda otra certeza: la labor docente es un trabajo profesional (no hay que olvidarlo) que requiere de un saber especializado que no puede ser reemplazado por "padres voluntariosos" o simples "voluntarios" (como si la simple "v" de vocación y voluntad alcanzaran), tal como quiso implementar hace pocos años la exgobernadora de la Provincia de Buenos Aires frente a la suspensión de clases por conflictos gremiales (TÉLAM, 28/02/17).

La continua contraposición entre profesión y vocación que tensiona la identidad y el oficio docente (DUBET, 2013; TENTI FANFANI, 2007) hoy se deja traslucir en diferentes discursos que socaban el reconocimiento social de los maestros y profesores. Aquí recuperamos una compilación de audios animados del canal de Youtube "Gente Rota"2 donde se escuchan algunas voces de madres que se quejan

\footnotetext{
${ }^{1}$ El escueto idioma ingles nos brinda una representación gráfica-morfológica del trastrocamiento del cronosistema social y su relación imbricada con las temporalidades escolares y laborales. Solo tres palabras distancia semánticamente dos actividades que hoy se ven obligadas a convivir a tiempo completo: homeworking

${ }^{2}$ Canal de youtube: https://www.youtube.com/channel/UCqzSuSBqST6kuWqYhorZ1tw. El humor constituye un calidoscopio interesante para la crítica social. Los audios utilizados fueron publicados durante el mes de abril, fecha en la cual se escribió el presente ensayo.
} 
de las actividades que proponen virtualmente las y los docentes y el trabajo que implica gestionar la temporalidad escolar: "Se hacen ahora los trabajadores”, “¿me van a dar parte de su sueldo?”. Una vez más, el trabajo que lxs docentes hacen cuando no están frente a curso (muchas veces no remunerado) queda doblemente invisibilizado (por el no reconocimiento social de dicho tiempo y su no remuneración). En el contexto actual podríamos mencionar un tercer aspecto de esta invisibilización: el tiempo que a los profesores les está llevando "reconvertir" sus clases presenciales a la modalidad virtual. Muchas veces se piensa como una mera trasferencia, un "copiar y pegar” que no requiere de cierta transposición didáctica. Esa "adaptación" de las clases no solo requiere un tiempo para pensar nuevamente toda la clase, sino que también conlleva un tiempo de aprendizaje de los maestros en la apropiación de las herramientas informáticas; quienes en muchos casos no contaban con dicho saber, una expresión más de la desigualdad en la formación y el ejercicio docente.

\section{EL FORMATO ESCOLAR OMNIPRESENTE}

El contexto de incertidumbre y desconcierto actual nos propicia otra certeza: el éxito del, muchas veces considerado perimido, formato escolar. Tanto Dubet (2013) como Vincent, Lahire y Thin (2001) afirman que las críticas del programa institucional, en un caso, o la forma escolar, en el otro, es producto del éxito y crecimiento que ha tenido ese formato a lo largo de la historia. Consideramos que se trata de un éxito (no de eficacia) objetivo, simbólico y subjetivo.

Nos referimos a un éxito objetivo porque dicha forma escolar hoy hegemoniza el funcionamiento de las escuelas y el cierre de sus edificios materializa la existencia de algo que hoy está enclenque. Le falta uno de sus pilares, esa arquitectura escolar que sostenía una determinada temporalidad. Pero no está desaparecida, varios de sus pirales están intactos.

Hablamos de éxito simbólico porque pareciera ser la forma socialmente más legitimada a la hora de dar rienda, no tan suelta, a la "continuidad pedagógica”. Toda forma alternativa, puede generar la sospecha de no ser continuidad pedagógica y, por ende, socavar el oficio docente.

Hablamos de éxito subjetivo porque en este período excepcional volvemos a recrear, sin querer queriendo, el formato escolar: enviamos videos de 40 minutos o más, tomamos lista por Zoom, dividimos en espacios de teóricos y de prácticos las aulas virtuales universitarias, etc. Es lógico, por un lado, en tiempos de incertidumbres necesitamos apoyarnos en certezas, y el formato escolar nos brinda una de ellas, aunque seamos conscientes de su pérdida de eficacia pedagógica. Por otro lado, dicho formato estructuró nuestra forma de ver y pensar el mundo y actuamos en consecuencia. No se trata, como muchos creen, de hacer lo que es más fácil, ¿saben cuánto tiempo lleva pensar, realizar y subir un video de 40 minutos?

Esto no solo le pasa a las y los docentes, sino también a los padres. Interpelados ahora a cumplir su parte del nuevo contrato pedagógico para sostener la virtual continuidad pedagógica, algunos intentan recrear el cronosistema escolar al interior de la arquitectura hogareña. No solo el espacio áulico ingresa a los diferentes domicilios, sino que la casa es el aula. Nuevamente recurrimos al video-audio de "Gente Rota”. Una de las madres idea una estrategia: “va a hacer lo mismo que el horario labo... del colegio”. No es casualidad que lo primero que se le venga a la cabeza es el "horario laboral”. Continua: "A la una nos sentamos tipo escuela, yo le doy la consigna, tengo los horarios de los recreos y que haga lo que haga”. Es decir, la estrategia es replicar la temporalidad escolar en la casa, incluso con los mismos recreos.

\section{VIRTUALIDAD, AUTORREGULACIÓN Y DESIGUALDAD}

La virtualidad escolar forzada trae un plus de exigencia para para las y los estudiantes. Requiere de una mayor autonomía y autorregulación individual. Desde ya que los gradientes van a variar en función del nivel educativo al que asistan. No es lo mismo reconstruir la temporalidad escolar de niñas y niños que asisten al prescolar o de jóvenes que cursan el nivel secundario. Los primeros van a necesitar una presencia más intensiva de los padres que los segundos. Pero cada uno en su respectivo nivel, va a requerir de un mayor autocontrol para responder a las exigencias de la educación virtual. 
Ya no hay un edificio, un aula, hay que recrearse el propio espacio. En aquellos casos en los cuales no se den clases sincrónicas ni siquiera hay un horario de llegada, un tarde, bloques de horas, recreos, hay que autoadministrarse el tiempo. Es decir, cada uno individualmente tiene que gestionarse el orden escolar (vale tanto para alumnos como docentes). Para ello se requiere de una estructura psíquica con un alto grado de autocontrol (ELIAS, 2011). Y esto no se inculca con discursos morales como "tenés que estudiar", "la escuela es importante"; sino con condiciones sociales que posibiliten estudiar. El año pasado una estudiante de secundario de la CABA en Argentina nos contaba que por estar ayudando a sus padres en el taller de costura informal no había hecho una tarea y por eso la estaba haciendo en los recreos. Le preguntamos por qué no les contó a los padres que tenía una tarea pendiente. Respondió que "se los había dicho pero que a la vez sentía que tenía que ayudarlos porque, aparte que es su responsabilidad, tuvo un gran lapso de tiempo para hacer la tarea y no la hizo"; por eso sintió que "tenía que joderse". $\mathrm{Al}$ final de su respuesta rio.

Nos preguntamos cómo estará atravesando hoy la virtualidad pedagógica esta estudiante. Sabemos por conversaciones que mantuvimos con directivos y docentes de esa escuela que el contexto está muy difícil. Muchos padres perdieron sus trabajos, varios alumnos tienen problemas de conexión para seguir las clases y cada vez más familias van a la escuela a pedir la vianda de alimentos. La situación tampoco es nueva. Recordamos que cuando le preguntamos aquella estudiante qué era lo que más le gustaba de la escuela respondió visceralmente: la comida. A ella no le faltaba autodisciplina o comprender la importancia de estudiar, le faltaba, tiempo, espacio y un plato de comida.

\section{CONSIDERACIONES FINALES}

Como podemos observar a lo largo de este pequeño ensayo, en este contexto de pandemia, la desigualdad social, trasmutada en desigualdad educativa mediante la continuidad pedagógica, se hace más presente y encarnizada.

Aquellos estudiantes de sectores económicamente más acomodados son quienes mayores posibilidades tienen de autogerenciarse el tiempo escolar. A su vez, en varias ocaciones son los que despotrican contra el formato escolar obsoleto. Sin embargo, son los que generalmente asisten a escuelas con alta dotación tecnológica y cuentan con la posibilidad de recrear lo más fielmente posible la temporalidad escolar que critican. Tienen que levantarse al mismo horario que cuando van a la escuela. Tienen clases sincrónicas, los profesores le toman lista y no se pueden ir del aula virtual (su habitación) hasta que termine la clase.

Justamente aquellos estudiantes que cuentan con menos condiciones objetivas de posibilidad para autorregularse son quienes más las necesitan porque el orden espaciotemporal de la escuela (que los sustraía durante unas horas de dichas condiciones desfavorables) ahora está interrumpido. Sin embargo, la continuidad pedagógica virtualizada, con todas sus dificultades, busca no perder el contacto con estxs estudiantes y mantener de alguna forma algún tipo de vínculo pedagógico. A veces no puede ser un ida y vuelta y solo es la emisión de un mensaje. Hay docentes que dan las clases por radio, envían fotocopias o pendrives a los parajes rurales, se contactan con el vecino de unx estudiante que llegó al pueblo a comprar algo. La idea es que el mensaje llegue aunque no pueda haber una respuesta en línea, que ese o esa estudiante sepa que del otro lado hay alguien al que puedan recurrir si lo llegaran a necesitar y que va a estar esperándolos cuando la cuarentena termine.

Concluimos que la pandemia visibilizó la potencialidad de la materialidad de la escuela. Situación que muchas veces es menoscabada, por ejemplo, cuando se la desprestigia o se la invisibilizada porque estamos acostumbrados a ese lugar que ocupa habitualmente. La actual clausura de los edificios escolares nos ayuda a "tocar" a la escuela como hecho social.

La reconfiguración de la temporalidad escolar trastocó los estilos de vida familiares de toda la comunidad educativa (tanto de docentes como de estudiantes). La educación en la virtualidad está reconfigurando el trabajo de enseñar y de aprender, desde el tiempo que nos implica apropiarnos de las herramientas tecnológicas, hasta cómo autorregular las demandas y posibilidades. 
Es sabido que esta experiencia excepcional nos va a dejar diferentes aprendizajes. Desde ya tendrá un impacto sobre el rol y el funcionamiento de la escuela, pero también sobre el vínculo entre la escuela, el hogar y la familia. La evaluación de la continuidad pedagógica podremos hacerla una vez que se retornen las clases. Ahí veremos si permitió continuidad, si sostuvo vínculos o si resultó un "como sí”.

\section{REFERÊNCIAS}

DE LA CRUZ FLORES, G. El hogar y la escuela: lógicas en tensión ante la COVID-19. In: IISUE (Ed.). . Educación y pandemia. Una visión académica. Ciudad de México: UNAM, 2020. p. 39-46. Disponible en: http://www.iisue.unam.mx/nosotros/covid/educacion-y-pandemia. Acceso el 25 de may. de 2020.

DUBET, F. El declive de la institución: profesiones, sujetos e individuos en la modernidad. Barcelona: Gedisa, 2013.

DURKHEIM, É. Las reglas del método sociológico. Torrejón de Ardoz, Madrid: Akal, 1987.

ELIAS, N. El proceso de la civilización. Investigaciones sociogenéticas y psicogenéticas. 3. ed. México: Fondo De Cultura Económica, 2011.

ESCOLANO, A. Tiempos y espacios para la escuela: ensayos históricos. Madrid: Biblioteca Nueva, 2000.

MARTÍN-BARBERO, J. Reconfiguraciones de la comunicación entre escuela y sociedad. In: TENTI FANFANI, E. (Ed.). Nuevos temas en la agenda de política educativa. Buenos Aires: Organización de las Naciones Unidas para la Educación, la Ciencia y la Cultura, Instituto Internacional de Planeamiento de la Educación, Sede Regional Buenos Aires: Siglo Veintiuno Editores, 2008. p. 65-99. .

SARAVÍ, G. A. Juventudes fragmentadas: socialización, clase y cultura en la construcción de la desigualdad. 1. ed. México, D.F: FLACSO México : CIESAS, 2015.

TELAM, Vidal anunció que convocará “voluntarios” si el paro docente se concreta, Política, 28/02/2017. Disponible en: https://www.telam.com.ar/notas/201702/181049-vidal-anuncio-queconvocara-voluntarios-si-el-paro-docente-se-concreta.html. Acceso el 07 de may. de 2020.

TENTI FANFANI, E. (ED.). El oficio de docente: vocación, trabajo y profesión en el Siglo XXI. Buenos Aires: Siglo XXI Editores Argentina, 2007.

VINCENT, G.; LAHIRE, B.; THIN, D. Sobre a História e a Teoria da Forma Escolar. Educação em Revista, n. 33, p. 7-47, 2001. 